ORIGINALES

\title{
Terapia grupal en prevención de recaídas del alcoholismo frente a seguimiento ambulatorio habitual
}

\section{Group psychotherapy for prevention of relapses in alcoholism compared to standard outpatient follow-up}

Pilar Calvo Estopiñán*; Alfonso Pérez Poza**; Pilar Sacristán Martín ${ }^{* *}$; Cristina Paricio GARCIA $^{* \star \star *}$
*Hospital Universitario Miguel Servet, Zaragoza. Médico Residente Psiquiatría.

**Hospital Universitario Miguel Servet, Zaragoza. Médico Adjunto Psiquiatría.

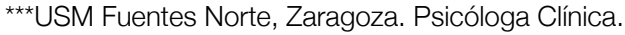

${ }^{\star \star \star \star}$ USM Fuentes Norte, Zaragoza. DUE Psiquiatría.

Enviar correspondencia a:

Pilar Calvo Estopiñán. C/ Monasterio San Juan de la Pena, $\mathrm{n}^{\circ} 1$, Esc 3, $1^{\circ}$ B. Cuarte de Huerva. C.P. 50410. Zaragoza.

E-mail: piluca@seny.org. Tfno: 652898825

\section{RESUMEN}

Introducción: La prevención de recaidas es el punto central y más original de la terapéutica de las adicciones. En este sentido hay estudios que avalan la eficacia de la terapia grupal. Objetivos: comparar la terapia grupal de prevención de recaídas con el seguimiento habitual de pacientes con abuso-dependencia alcohólica. Metodología: se asignaron 14 pacientes al grupo de prevención de recaídas y 10 pacientes realizaron el control ambulatorio habitual. Se recogieron el número de recaidas durante el tiempo que duró la terapia grupal y al año. Resultados: Encontramos diferencias estadisticamente significativas $(\chi 2(2)=7,95 ; p=0,019)$ en cuanto a las tasas de abstinencia y recaida entre ambos grupos en el seguimiento a 12 meses. Conclusiones: la terapia grupal de prevención de recaída es fácil de aplicar en la consulta externa, eficiente y obtiene mejores tasas de abstinencia en el seguimiento al año, que el tratamiento ambulatorio habitual.

Palabras clave: alcoholismo, psicoterapia grupal, recaida, prevención.

\section{ABSTRACT}

Introduction: Relapse Prevention (RP) is the central and most original focus in the treatment of addictions. Several studies support the effectiveness of group therapy in RP. Aims: To compare group psychotherapy for RP with standard follow-up of alcohol-abuse/ dependence patients. Method: Fourteen patients were assigned to the relapse prevention group and another ten patients received the standard outpatient monitoring. Numbers of relapses during the group therapy and one year later were recorded. Results: We found statistically significant differences $(\chi 2(2)=7.95 ; p=0.019)$ between the two groups for rates of abstinence and relapse at the twelve-month follow-up. Conclusions: Group Therapy for Relapse Prevention is effective, easy to apply in the outpatient context and obtains better rates of abstinence than standard outpatient treatment at the one-year follow-up.

Key words: alcoholism, group psychotherapy, relapse, prevention. 


\section{INTRODUCCIÓN}

$\mathrm{D}$ entro del grupo de las drogodependencias, el consumo perjudicial de alcohol (CIE-10)' ${ }^{1}$, abuso y dependencia de alcohol (DSM-IV-TR)2 son los trastornos más prevalentes.

El tratamiento de la dependencia alcoholica es complejo, dinámico y largo, debe ser entendido como un proceso ${ }^{3,4}$, 5. El esquema terapéutico básico en el alcoholismo es: 1. Desintoxicación, 2. Deshabituación, 3. Mantenimiento de la abstinencia, con especial atención a la prevención de recaídas, y 4. Recuperación y reinserción social

La prevención de recaídas (PR) es el punto central y más original de la terapéutica de las adicciones. Vaillant (1983) realizó un seguimiento de 685 sujetos tratados por problemas relacionados con el alcohol y observó que a los 2 años de tratamiento el 63\% había vuelto a su conducta adictiva, y al final del seguimiento de 8 años de duración, el $95 \%$ de la muestra habia reanudado su consumo de alcohol ocasionalmente.

El alcoholismo es una enfermedad crónica en la que la recuperación no siempre es completa ni duradera. Hoy día conocemos mejor el modo de prevenir recaídas. La prevención de recaídas se define como "el proceso de iniciar y mantener la abstinencia de alcohol o de otra sustancia, acompañado de modificaciones intra e interpersonales"6. Así pues, la PR es un programa de autocontrol diseñado con el fin de ayudar a los individuos a anticipar y afrontar los problemas de recaída en el cambio de las conductas adictivas. Basada en principios de la psicología de la salud y en la teoría social cognoscitiva de Bandura ${ }^{7}$, la PR combina los procedimientos conductuales de entrenamiento en habilidades, la terapia cognitiva y el reequilibrio del estilo de vida.

La fuerza básica contra el alcoholismo radica en la psicoterapia de grupo y las medidas psicosociales. La Terapia de Grupo es una herramienta terapéutica utilizada ampliamente en el tratamiento del alcoholismo con diferentes tipos de programas y procedimientos (grupos de autoayuda, grupos de familias, psicoeducativos, introspectivos, motivacionales, de prevención de recaídas... $)^{8}$.

El grupo permite aceptar y entender la enfermedad, da herramientas para la autonomía del paciente y prepara al individuo para situaciones de riesgo, asi mismo aumenta la motivación para la abstinencia y disminuye la tendencia a la negación y da respuesta a la necesidad de adaptación social ${ }^{8,9}$. Se potencia la autoeficacia del paciente, ayudándole a reconocer las situaciones de riesgo y a utilizar actitudes alternativas evitando nuevos consumos ${ }^{10}$.

Existe una vasta literatura que defiende la terapia de grupo en alcohólicos bien en forma de grupo de discusión, de orientación psicodinámica, cognitivo-conductual o interaccional ${ }^{11}, 12,13,14,15,16$.

Colli señala que la terapia de grupo en alcohólicos a largo término ofrece resultados efectivos por la presencia de determinados factores curativos de grupo como: altruismo, comprensión de sí-mismo, revalidación de la familia, aprendizaje interno y cohesión grupa $1^{17}$.

El estudio de Monras y Gual ${ }^{18}$ confirma, tras el examen y seguimiento de 329 alcohólicos en terapia de grupo la utilidad de esta herramienta terapéutica en el tratamiento de la enfermedad en la que se señala como crítica la adherencia al tratamiento como principal predictor del éxito del mismo. Los mismos autores ${ }^{19,20}$ han confirmado recientemente la efectividad de las técnicas grupales en un ensayo aleatorizado, y la persistencia de sus efectos a largo plazo.

Sin embargo, a pesar de que los terapeutas y pacientes que han participado en terapia de grupo la consideran útil y eficiente, muchos estudios han señalado lo difícil que es evaluar los procesos de terapia de grupo y su eficacia. Asimismo la comparación entre terapia individual y terapia de grupo es complicada y los resultados permanecen poco claros. Los estudios que evalúan la terapia de grupo han mostrado que los pacientes que están en psicoterapia grupal mejoran su grado de compromiso, tienen tasas más altas de abstinencia mejoran en un número de variables relacionadas con la calidad de vida, mejoran su transición de tratamiento interno a tratamiento ambulatorio y son más proclives a volver a las citas de seguimiento. Debe apuntarse, sin embargo, que la extrapolación de estos resultados es difícil, ya que se han obtenido con diferentes tipos de orientaciones, objetivos, técnicas y liderazgos ${ }^{21}$.

El objetivo de nuestro trabajo es comparar las tasas de abstinencia alcohólica obtenidas en el grupo de prevención de recaídas frente al grupo que realiza seguimiento ambulatorio habitual.

Así mismo, en el presente artículo queremos dar a conocer la estructura formal y de contenidos de nuestro grupo de prevención de recaídas en el alcoholismo, las características clínicas y sociodemográficas de los miembros que lo componen y los resultados obtenidos.

\section{MÉTODOS}

Se trata de un estudio descriptivo longitudinal prospectivo con grupo control. La muestra está compuesta por pacientes con diagnóstico de abuso o dependencia a alcohol, abstinentes por lo menos desde hace 1 mes. La existencia de comorbilidad psiquiátrica no es criterio de exclusión. Los pacientes pueden estar tomando tratamiento psicofarmacológico prescrito por su psiquiatra. Se hicieron dos grupos de comparación, uno de $\mathrm{N}=10$ que recibe tratamiento habitual en su USM mediante visitas programadas con su psiquiatra y otro grupo de terapia $(\mathrm{N}=14)$ que acude además de a las visitas programadas con su psiquiatra, al grupo de prevención de recaídas. La asignación a uno u otro grupo se realizó de forma aleatoria.

Establecemos las diferencias entre reanudación del consumo o recidiva y recaída según el criterio de Volpicelli ${ }^{22}$, 
que fijó como criterio de "recaída" el consumo de $>5$ consumiciones por ocasión de consumo ó $>5$ días de ingestión de alcohol a la semana. Análogamente, como criterio de "recidiva o reanudación del consumo", se consideró la ingestión de 1 a 5 consumiciones de alcohol por ocasión de consumo o entre 1 y 5 días bebiendo a la semana.

Para el tratamiento de los datos estadísticos usamos el paquete estadístico SPSS 12.0 plus realizando un análisis descriptivo de las variables del estudio, así como comparaciones entre datos cualitativos mediante el test de Chi-cuadrado.

\section{Características del grupo de terapia de prevención de recaídas de alcoholismo}

1. Se lleva a cabo en medio ambulatorio con pacientes no hospitalizados.

2. Es un grupo cerrado, aquellos en que los participantes se definen al comienzo de la terapia y no se modifican hasta que ésta termina, salvo por circunstancias especiales como el abandono.

3. Grupo heterogéneo: es decir, hay diferencias tanto clínicas (abuso/dependencia alcohólica vs patología dual) como sociodemográficas (sexo, edad) entre los miembros. El grupo está, formado por 14 personas, 10 hombres y 4 mujeres con edades comprendidas entre los 23 y los 65 años.

Al inicio de la primera sesión los pacientes firman un contrato terapéutico de normas de funcionamiento del grupo.

\section{Marco espacial}

El lugar elegido para realizar las reuniones es una amplia sala de grupos ubicada en el mismo centro de salud que la propia USM a la que pertenecen los pacientes. Se trata de un espacio amplio, que proporciona intimidad, libre de posibles interrupciones para evitar así posibles distracciones. Las sillas son dispuestas en círculo.

\section{Marco temporal}

Las sesiones se llevan a cabo cada 15 dias aproximadamente, durante un periodo de tres meses (iniciándose el grupo el 31 de octubre de 2006 y finalizando el 30 de Enero de 2007).

\section{Contenido y estructuración de las sesiones}

Antes de cada sesión se hace test de alcoholemia en aire espirado. Si es positivo el paciente no puede acudir a esa sesión ni a la siguiente.
La terapia tiene una duración de 7 sesiones, cada una de ellas dedicada a uno de los módulos que a continuación se detallan:

1. Matriz de decisiones y etapas de motivación para el cambio.

2. Repercusiones para la salud.

3. Prevención de recaídas.

4. Situaciones de riesgo.

5. Solución de problemas.

6. Estrategias de intervención y de afrontamiento I: Contrato terapéutico, control de estímulos, habilidades sociales, revisión de la matriz de decisiones.

7. Estrategias de intervención y de afrontamiento II: Detección de antecedentes de riesgo, apoyo sociofamiliar.

Cada sesión o módulo tiene una duración de 1 hora y media, distribuyéndose el tiempo de la siguiente manera en cada uno de ellos:

a. En el primer cuarto de hora/media hora uno de los terapeutas hace la introducción del tema a trabajar en esa sesión. Intentamos que está primera parte tenga función psicoeducativa pero a la vez procuramos que sea bastante dinámica, interaccionando con los pacientes en todo momento.

b. El resto del tiempo se dedica al grupo propiamente dicho, donde cada paciente habla de sus experiencias personales, de sus sentimientos, temores, dudas... participando en él un terapeuta principal y dos co-terapeutas.

c. Una vez finalizado el grupo, la enfermera de la USM realiza 20 minutos de relajación siguiendo el método de Jacobson. Durante este tiempo el resto del equipo realiza una sesión postgrupo analizando lo sucedido en el módulo terapéutico de ese día.

\section{Terapeutas}

Las sesiones están dirigidas por un terapeuta y dos coterapeutas (psiquiatra, psicóloga y residente de psiquiatría). Los terapeutas tienen una función directiva y facilitadota de la comunicación entre sus miembros, evitando en la medida de lo posible, los silencios y el bloqueo emocional de la comunicación entre las sesiones. La terapia de relajación la realiza la enfermera de salud mental del centro.

Este grupo de prevención de recaídas en el alcoholismo se llevó a cabo dentro del programa FOCUSS, el cual es un método de formación para profesionales del S.S.P.A. que consiste en facilitar el acceso a una formación práctica, organizada, de calidad y evaluada. En él participaban como alumnas 3 enfermeras de la unidad de hospitalización breve 
de Psiquiatría de otro Hospital de la Comunidad Autónoma que tenían el proyecto de poner en marcha un grupo de similares características en su lugar de trabajo.

Este tipo de programas formativos sigue desarrollándose actualmente en nuestro hospital.

\section{RESULTADOS}

El grupo de prevención de recaídas está formado por 10 hombres y 4 mujeres, con edades comprendidas entre los 23 y 65 años, siendo la edad media de 49,43 años (D. T. = 11,39). La mayoría de ellos están casados (50\%). El 64,3\% tienen hijos, el resto no tiene descendencia. Desde el punto de vista laboral, sólo el 14,3\% está en activo. Según la comorbilidad psiquiátrica se distribuyen de la siguiente manera: $35,7 \%$ no tiene otro diagnóstico psiquiátrico comórbido, el 50\% tiene un diagnóstico psiquiátrico comórbido, el 7,1\% tiene dos diagnósticos psiquiátricos comórbidos y otro 7,1\% tiene tres. El resto de características sociodemográficas, clínicas y los problemas añadidos derivados del consumo de alcohol, tanto del grupo de PR como de los pacientes en seguimiento ambulatorio se muestran en la tabla 1. Los diagnósticos psiquiátricos comórbidos que encontramos aparecen reflejados en la tabla 2 .

Durante los meses que duró la terapia grupal, los resultados en el grupo de terapia fueron los siguientes: el 64,3\% mantuvo una abstinencia total en el consumo de alcohol, el 28,57\% tuvo alguna recidiva sin llegar a la recaída, y únicamente una paciente (lo que supone un 7,13\%) abandonó el grupo por recaída completa. Mientras que en el grupo control, aquel que únicamente recibe visitas programadas en su USM, durante la terapia sólo el 50\% conseguian mantener una abstinencia completa, el 20\% tuvieron alguna recidiva y el 30\% alguna recaída (Figura 1), no siendo estos resultados estadísticamente significativos $\left(\chi^{2}(2)=0,98 ; p=0,613\right)$. Sin embargo, en el seguimiento a 12 meses encontramos diferencias estadísticamente significativas $\left(\chi^{2}(2)=7,95 ; p=0,019\right)$ entre ambos grupos, fundamentalmente en cuanto a tasas de abstinencia y recaída; observamos en el grupo de terapia que el $64,3 \%$ se mantenía abstinente al cabo del año, el 21,4\% tuvo alguna recidiva y el $14,3 \%$ recayó en el consumo. Sin embargo en el grupo control, únicamente el 10\% mantuvo la abstinencia durante los 12 meses, el 30\% tuvo alguna recidiva y el 60\% recayó (Figura 2).

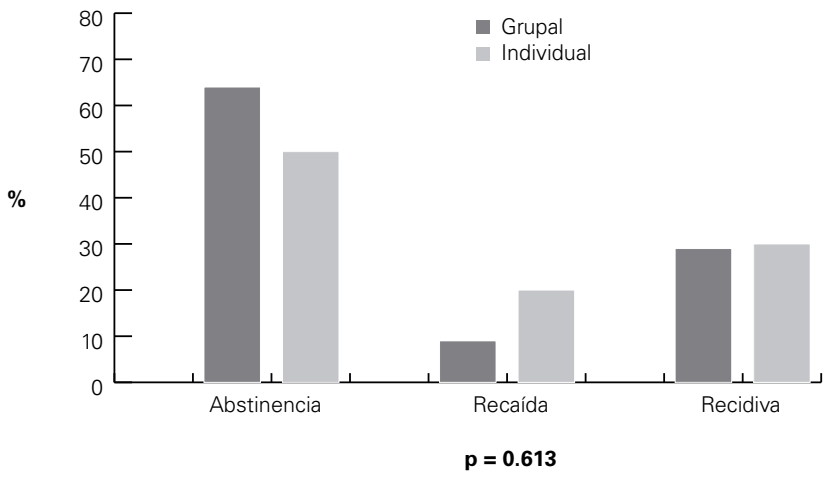

Figura 1. Seguimiento de la evolución durante el tiempo que duró la terapia grupal.
Tabla 1. Características sociodemográficas, clínicas y problemas derivados del consumo de alcohol del grupo de prevención de recaídas y del grupo control.

\begin{tabular}{|c|c|c|}
\hline Característica & $\begin{array}{l}\text { Pacientes en Terapia } \\
\text { Grupal de } \\
\text { Prevención de Recaídas } \\
(n=14)\end{array}$ & $\begin{array}{c}\text { Pacientes en } \\
\text { seguimiento } \\
\text { ambulatorio individual } \\
\quad(n=10)\end{array}$ \\
\hline Edad & $49,43 \pm 11,393$ & $37,6 \pm 13,713$ \\
\hline $\begin{array}{l}\text { Sexo \% (n) } \\
\text { Hombres } \\
\text { Mujeres }\end{array}$ & $\begin{array}{c}71,4(10) \\
28,6(4)\end{array}$ & $\begin{array}{l}70(7) \\
30(3)\end{array}$ \\
\hline $\begin{array}{l}\text { Estado civil \% (n) } \\
\text { Soltero } \\
\text { Casado } \\
\text { Separado/divorciado } \\
\text { Viudo }\end{array}$ & $\begin{array}{l}28,6(4) \\
50,0(7) \\
21,4(3) \\
0,0(0)\end{array}$ & $\begin{array}{l}40(4) \\
60(6) \\
0(0) \\
0(0)\end{array}$ \\
\hline $\begin{array}{l}\text { Situación laboral \% (n) } \\
\text { Activo } \\
\text { Parado }\end{array}$ & $\begin{array}{c}14,3(2) \\
85,7(12)\end{array}$ & $\begin{array}{l}70(7) \\
30(3)\end{array}$ \\
\hline $\begin{array}{l}\text { Antecedentes familiares \% (n) } \\
\text { Psiquiátricos } \\
\text { Alcoholismo } \\
\text { Otras drogodependencias }\end{array}$ & $\begin{array}{l}21,4(3) \\
50,0(7) \\
14,3(2)\end{array}$ & $\begin{array}{l}70(7) \\
40(4) \\
10(1)\end{array}$ \\
\hline $\begin{array}{l}\text { Problemas añadidos \% (n) } \\
\text { Médicos } \\
\text { Laborales } \\
\text { Familiares } \\
\text { Legales } \\
\text { No problemas }\end{array}$ & $\begin{array}{l}78,6(11) \\
21,4(3) \\
42,8(6) \\
7,1(1) \\
7,1(1)\end{array}$ & $\begin{array}{l}10(1) \\
10(1) \\
70(7) \\
0(0) \\
20(2)\end{array}$ \\
\hline $\begin{array}{l}\text { Comorbilidad psiquiátrica } \\
\% \text { (n) } \\
\text { NO } \\
\text { SI }\end{array}$ & $\begin{array}{l}35,7(5) \\
64,3(9)\end{array}$ & $\begin{array}{l}30(3) \\
70(7)\end{array}$ \\
\hline
\end{tabular}

Tabla 2. Diagnósticos psiquiátricos comórbidos encontrados en la muestra.

\begin{tabular}{|c|c|}
\hline $\begin{array}{l}\text { Pacientes en Terapia Grupal } \\
\text { de Prevención de Recaídas } \\
\qquad(n=14) \%(n)\end{array}$ & $\begin{array}{l}\text { Pacientes en seguimiento } \\
\text { ambulatorio individual } \\
(n=10) \%(n)\end{array}$ \\
\hline Deterioro cognitivo 7,1 (1) & Retraso Mental leve 10 (1) \\
\hline Ludopatía 7,1 (1) & Trastorno Depresivo 10 (1) \\
\hline Trastorno de Ansiedad no especificada 7,1 (1) & Trastorno de Ansiedad Generalizada 10 (1) \\
\hline Trastorno de Personalidad 14,3 (2) & Trastorno de Personalidad 10 (1) \\
\hline Trastorno Adaptativo 21,4 (3) & Trastorno Adaptativo 20 (2) \\
\hline Trastorno Bipolar 21,4 (3) & Abuso otros tóxicos 20 (2) \\
\hline
\end{tabular}

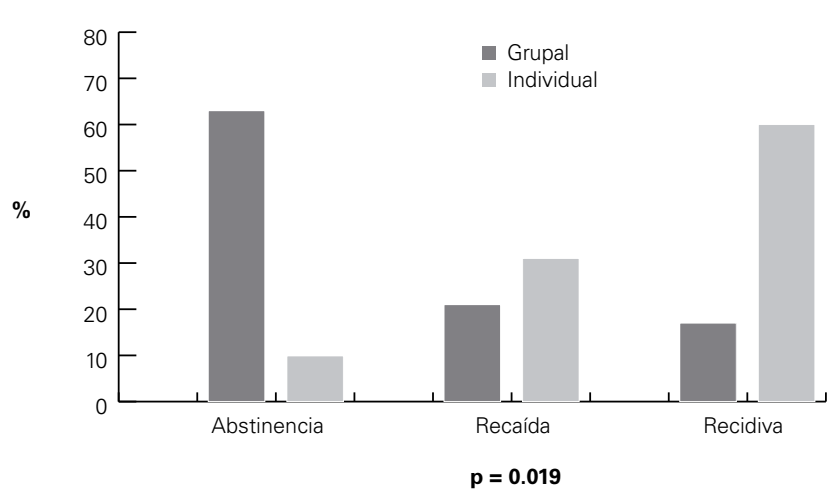

Figura 2. Seguimiento de la evolución al año. 


\section{DISCUSIÓN}

El conjunto de las psicoterapias de grupo abarca todas aquellas técnicas terapéuticas en las cuáles un conjunto de personas con una misma patología o que comparten una serie de características comunes susceptibles de tratamiento, son seleccionadas y reunidas en un grupo dirigido por un terapeuta con el objetivo de que dichas personas se presten ayuda mutua. Pero además la psicoterapia grupal tiene muchas propiedades únicas: proporciona relaciones interpersonales, aporta cohesión a los miembros, facilita la generalización de problemas, etc.

En lo tocante a la relevancia de la psicoterapia de grupo es importante recalcar que su eficacia es igual o superior a la individual. Se está produciendo una generalización en el empleo de estas terapias.

Consideramos que nuestro esquema de terapia grupal en prevención de recaídas en el alcoholismo es fácil de reproducir pudiéndose llevar a cabo en el propio centro de salud, puede ser dirigido por cualquier miembro del equipo de salud mental (psiquiatra, psicólogo, enfermera).

La eficiencia, que incluye el balance económico, es evidente: un terapeuta atiende en un tiempo determinado (90 minutos en nuestro grupo) a varios pacientes, pudiendo así rentabilizar los espacios terapéuticos.

El 64,3\% de abstinencia que obtuvimos en el grupo de prevención de recaídas tanto durante la terapia como en el seguimiento al año puede parecer un resultado poco relevante a primera vista pero el abandono del consumo perjudicial de alcohol implica una considerable disminución de las complicaciones médicas, de las bajas laborales, accidentes, descompensaciones de la patología de base..., lo cual supone a su vez una considerable mejora en la calidad de vida para estos pacientes y una reducción importante de los gastos sanitarios derivados. Se puede concluir, además, que la eficacia de la terapia grupal perdura en el tiempo, ya que en el seguimiento al año de los pacientes sometidos a este tipo de intervención observamos mayores tasas de abstinencia y menores tasas de recaída que en el grupo control.

La principal limitación metodológica del estudio es el tamaño muestral pequeño, por ello debe ser considerado como un estudio piloto, siendo necesarios estudios con una serie de pacientes más amplia para poder confirmar los resultados hallados.

\section{REFERENCIAS}

1. OMS. CIE-10. Trastornos mentales y del comportamiento. Organización Mundial de la Salud. Madrid: Ed. Meditor; 1992.

2. APA. DSM IV TR. Manual diagnóstico y estadístico de los trastornos mentales. Barcelona: Ed. Masson; 2002.
3. Altisent Trota R, Pico Soler MV, Delgado Marroquin MT, Mosquera Nogueira J, Aubà i Llambrich J, Córdoba Garcia R. Protocolo de alcohol en atención primaria. FMC. 1997; 3: 5-31.

4. Galanter M. Tratamiento de los trastornos por abuso de sustancias de la American Psychiatric Press. Barcelona: Ed. Masson; 1997.

5. Hester RK, Miller WR. Handbook of alcoholism treatment approaches. Effective alternatives, 2nd ed. Boston, MA: Allyn \& Bacon; 1995.

6. Daley DC, Marlatt GA. En Lowinson J, Ruiz P, Millman R, Langrod J, editores. Relapse prevention: Cognitive and behavioral interventions. Comprehensive Textbook of Substance Abuse, 2nd edition. Baltimore: Williams and Wilkins; 1992. p. 533-542.

7. Bandura A. Self-efficacy: Toward a unifying theory of behavioral change. Psychol Rev 1977; 84: 191-215.

8. Kaplan HI, Sadock BI. Terapia de grupo. Madrid: Ed. Panamericana; 1996.

9. Vinagradov S, Yalom ID. Guía breve de psicoterapia de grupo. Barcelona: Ed. Paidós; 1996.

10. Bandura A. Self-efficacy mechanism in human agency. Am Psychol 1982; 37: 122-47.

11. Bogani E. Psicoterapia de grupo en el Alcoholismo. Drogalcohol 1984; 1: 65-77.

12. Golden S, Halliday K, Khantzian E, McAuliffe W. Terapia de grupo dinámica para pacientes adictos a sustancias: Reconceptuación. En: Alonso A, Zwiller H, editores. Psicoterapia de Grupo en la práctica clínica. México D. F.: Comp. Ed Manual Moderno; 1995.

13. Hernández Boado M. Estructuración de un centro de Asistencia para la Rehabilitación de enfermos y enfermas alcohólicas. Revista Española de Drogodependencias 1989; 14: 249-55.

14. Monrás M, Freixa N, Ortega L, Lligoña A, Mondón S, Gual A. Eficacia de la terapia de grupo para alcohólicos. Resultados de un ensayo clínico controlado. Med Clin 2000; 115: 126-31.

15. Martínez A, Fernández JM. Grupos de Psicoterapia extrahospitalaria. En: Bobes J, González-Quirós P, Portilla P. Treinta años de alcohol y otras drogas en Asturias. Oviedo: Gofer; 1996.

16. Cuadrado P. Evolución de la dependencia alcohólica en tratamiento. Factores predictivos en un seguimiento de 5 a 7 años. Adicciones 1998; 10: 335-41.

17. Colli Alonso M, Zaldivar Pérez D. Factores Curativos en psicoterapia de grupo: su evaluación y análisis en una muestra de alcohólicos rehabilitados. Adicciones 2002; 14: 381-91.

18. Monras M, Gual A. Attrition in Group Therapy with alcoholics: a Survival Analysis. Drug Alcohol Rev 2000; 19: 55-63.

19. Monras M, Freixa N, Ortega L, Lligoña A, Mondon S, Gual A. Eficacia de la terapia de grupo para alcohólicos. Resultados de un ensayo clínico controlado. Med Clin 2000; 115: 126-31.

20. Lligoña $A$, Costa $S$, Gual $A$, Monrás M. Efficacité de la therapie de groupe chez le patient alcoolodépendant. Alcoologie et Addictologie 2000; 22: 401-07.

21. Martín ப. Tratamientos psicológicos. Adicciones 2002; 14: 40920.

22. Volpicelli JR, Alterman Al, Hayashida M, O'Brien CP. Naltrexone in the treatment of alcohol dependence. Arch Gen Psychiatry 1992; 49: 876-880. 


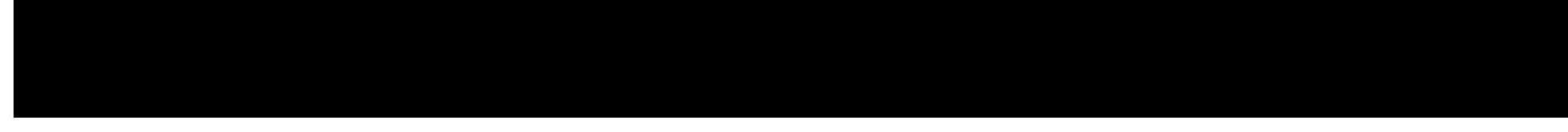

